improvement in pain in patients who received $\alpha$-adrenergic antagonists compared with those who received placebo; the pain associated with CP/CPPS is, therefore, not solely caused by the abnormal smooth muscle tone within the prostate gland and prostatic capsule and in the bladder-neck region. The authors speculate that $\alpha$-adrenergic-antagonist monotherapy might also improve the symptoms of CP/CPPS by causing relaxation of smooth muscle in the prostate stroma, if prostatic inflammation is present.

The authors suggest that additional therapies are needed to treat CP/CPPS effectively, and call for meta-analyses of high-quality, randomized, controlled trials.

Original article Yang G et al. (2006) The effect of $\alpha$-adrenergic antagonists in chronic prostatitis/chronic pelvic pain syndrome: a meta-analysis of randomized controlled trials. J Androl 27: $847-852$

\section{New potential biomarkers for interstitial cystitis}

Several promising biomarkers are known for this heterogeneous condition of unknown etiology, but levels of these markers correlate poorly with cystoscopic findings or treatment outcomes. Inflammation is, however, thought to have a pathogenic role in at least some subgroups of patients with interstitial cystitis.

Lamale and colleagues, therefore, measured levels of the inflammatory markers interleukin (IL)-6, histamine, and methylhistamine in $24 \mathrm{~h}$ urine samples from 40 women with interstitial cystitis (mean age 50.48 years) and 29 healthy control women (mean age 53.14 years). All participants avoided foods and medications that contained bioactive amines for the $24 \mathrm{~h}$ preceding urine collection. Levels of IL-6 and histamine were markedly higher in patients with interstitial cystitis than in controls, and there was also a similar trend in methylhistamine levels. The best combination of these markers for diagnosis of interstitial cystitis was IL-6 plus histamine (adjusted for urinary creatinine levels): when threshold values of $2.28 \mathrm{pg} / \mathrm{ml}$ for IL-6 and $288 \mu \mathrm{g} / \mathrm{g}$ for histamine were used, this combination of markers had $70.0 \%$ sensitivity, $72.4 \%$ specificity, and $77.8 \%$ positive and $63.6 \%$ negative predictive values for distinguishing patients from controls.
Despite these encouraging results, the authors note that antiproliferative factor has an even higher sensitivity and specificity (94\% and $95 \%$ ) than IL-6 plus histamine has for diagnosis of interstitial cystitis. They call for longitudinal studies to correlate levels of these biomarkers with disease activity, and speculate that combinations of biomarkers might be useful to confirm uncertain diagnoses of interstitial cystitis, or to select patients for additional therapies.

Original article Lamale LM et al. (2006) Interleukin-6, histamine, and methylhistamine as diagnostic markers for interstitial cystitis. Urology 68: 702-706

\section{Adjuvant radiotherapy benefits men with locally advanced prostate cancer}

In the US, 38-52\% of men who undergo radical prostatectomy for localized prostate cancer have extraprostatic disease. The optimal treatment for these patients remains unclear; adjuvant radiotherapy is known to delay biochemical recurrence and local progression, but whether survival is also improved remains unclear.

Thompson and colleagues' multicenter trial prospectively evaluated 425 men with stage pT3N0M0 prostate cancer after radical prostatectomy. Patients were randomly assigned to receive either 60-64Gy external-beam radiotherapy to the prostatic fossa $(n=214)$ or to usual care plus observation $(n=211)$, and were followed up for a median of 10.6 years. PSA-relapse-free survival and recurrence-free survival were markedly prolonged in the radiotherapy group, compared with the observation group (median 10.3 versus 3.1 years, and 13.8 versus 9.9 years, respectively). Compared with patients in the observation group, fewer patients in the radiotherapy group required hormonal therapy by 5 years after radical prostatectomy (10\% versus $21 \%$ ), but more experienced adverse effects, including rectal complications, urethral strictures and total urinary incontinence (23.8\% versus $11.9 \%)$. Far fewer metastases were seen than expected: the authors estimated metastasis-free survival in this cohort to be $78 \%$ at 13.2 years, which might have contributed to the lack of a statistically significant difference in metastasis-free survival between the two groups (median 14.7 versus 13.2 years). 